Original Article

\title{
Diagnostic Accuracy and Validity of Magnetic Resonance Imaging in the Detection of Specific Type of Brain Tumour
}

\author{
${ }^{*}$ Munshi $\mathrm{S}^{1}$, Ahmed F², Halder B ${ }^{3}$, Yousuf $\mathrm{A}^{4}$, Rahman $\mathrm{MM}^{5}$, Sarker $\mathrm{S}^{6}$
}

\begin{abstract}
Magnetic Resonance Imaging (MRI) is a widely accessible imaging technique for the detection of brain tumours and cancer, which are further confirmed by histopathological examination. Accurate detection of the tumours and its extent is very difficult. The present study attempted to evaluate the convenience of MRI in detection of different grades of astrocytomas, which are the most commonly occurring brain tumours. This cross-sectional study was conducted at the Department of Radiology and Imaging with the collaboration of Department of Neurosurgery and Department of Pathology at Sir Salimullah Medical College (SSMC \& MH), Dhaka from January 2013 to December 2013 for a period of one year. The study population was all the diagnosed cases of intracranial astrocytoma patients regardless of their age and sex. The studied included 48 brain tumour (astrocytoma) patients, ages between 13 and 69 years old. All cases having no contraindication for MRI underwent MR examination followed by histopathological examination of the postoperative resected tissues. The findings of the MRI and histopathological examination were compared to find out the test validity of the MRI findings of the different grades of astrocytoma's. The highest sensitivity was found in grade III astrocytoma (90.5\%) followed by grade II (85.7\%) grade IV (75.0\%) and grade I (60.0\%). The highest specificity
\end{abstract}

1. *Dr. Swati Munshi, MBBS. MD (Radiology \& Imaging) Specialist, Square Hospitals Ltd. Email: swatimahbub@hotmail.com

2. Dr. Farid Ahmed, Professor, Radiology and Imaging, Anowar Khan Modern Medical College. Ex. Head of the Dept. of Radiology \& Imaging, Sir Salimullah Medical College and Hospital, Dhaka.

3. Dr. Bibekananda Halder, Associate Professor, Radiology \& Imaging, Sir Salimullah Medical College and Hospital, Dhaka.

4. Dr. Abdullah Yousuf, Assistant Professor, National Institute of Neurosciences and Hospital, Dhaka.

5. Dr. Md. Mahbubur Rahman, Associate Professor, National Institute of Cardiovascular Diseases, Dhaka.

6. Dr. Snigdha Sarker, Assistant Professor, National Institute of Traumatology and Orthopedic Rehabilitation, Dhaka

*For Correspondence was found in grade I astrocytoma (97.7\%) followed by Grade III (96.3\%), grade IV (92.5\%) and grade II (91.5\%). The bighest accuracy was found in both grade I astrocytoma (93.7\%) and grade III (93.7\%) followed by grade II (92.5\%) and grade IV (89.6\%). As per the study findings it can be concluded that, MRI has a high diagnostic accuracy and validity for the detection of different grades of astrocytoma.

Key words: Magnetic Resonance Imaging; brain tumours; glioma; astrocytoma; validity test.

\section{INTRODUCTION}

Astrocytomas are the most common type of primary brain tumour within the group of brain tumours called gliomas. Plain radiograph was previously used in the past to detect such type oftumours. Cerebral angiography and pneumocephalography were also done; however, none of which was convincing. With the advent of CT and MRI there is revolutionary change in the detection of intracranial tumour. Diagnosing a brain tumor usually begins with magnetic resonance imaging (MRI), which if shows that, there is a tumor in the brain, the most common way to determine the type of brain tumor is to look at the results from a sample of tissue after a biopsy or surgery. MRI scan has made a significant impact on the differential diagnosis of intracranial tumours. ${ }^{3}$

MRI is more sensitive than CTfor detection of intra-cerebral astrocytomas and for signifying their extentand spread. Compared with CT, MRI offers greater contrast resolution,including greater sensitivity for the detection of subacute and chronic haemorrhage in association with tumours and other lesions of brain ${ }^{4}$. MRI also has lessionizing radiation. Delineation of posterior cranial fossa soft tissue anatomy is better visualized with MRI than CT as becauseMRI lacks beam-hardening artefact $^{5}$ and as it offers imaging from multiple planes $^{6}$, thus accuracy of lesion localization is enhanced. MRI is advanced with providing important information such as, contrast material enhancement, peritumoural oedema, distant tumour foci, haemorrhage, necrosis,mass effect and so on, which are all helpful in characterizing tumour aggressiveness and hence tumour grade. ${ }^{7}$ In this context,this study addresses the diagnostic precision of MRI as a brain investigation tool to assess astrocytoma diagnosis and the tumour staging. 


\section{METHODOLOGY}

This study was designed as cross sectional study which was carried out in the Department of Radiology and Imaging with the collaboration of Department of Neurosurgery and Department of Pathology at Sir Salimullah Medical College (SSMC \& MH), Dhaka from January 2013 to December 2013 for a period of one (1) year. A total number of 48 astrocytoma patients were evaluated by purposive sampling technique. Patients who were clinically suspected and CT scan diagnosed cases of intracranial astrocytomas referred to Radiology and Imaging department of Dhaka Medical college Hospital (DMCH) either from OPD or from indoor of DMCH for MRI of brain were included in this study. Prior to the commencement of this study, the research protocol was approved by the ethical committee (Local Ethical committee) of SSMC. All cases having no contraindication for MRI underwent MR examination. Patients were asked for or checked for any metallic or harmful. MR imaging was obtained with 0.3 Tesla machine (HITACHI). T1W image in axial, sagittal and coronal plane were obtained using short TR (500-800ms) and short TE (14-20ms). T2W image in axial and coronal plane were obtained using long TR (3500-4500ms) and long TE (80ms). FLAIR images were also taken. Contrast MRI studies using intravenous Gd-DTPA (Magnevist, $0.1 \mathrm{mmol} / \mathrm{Kg}$ ) with axial, coronal and sagittal T1Wscanwere performed in all cases. The average time of examination was 45 minutes but ranges from 30-90 minutes. Slice thickness was 5-6 mm with a field of view $230 \times 230 \mathrm{~mm}$ and pictures matrix was $256 \times 256$ or $192 \times 256$. The postoperative resected tissues were examined histopathological in the respective department. MRI scan findings were compared with histopathological reports. Then the collected reports were compared with findings of MRI. Data were collected using a preformed data collection sheet. Base line information was collected from the patient after exploration of different complaints and sign and symptoms. All information regarding clinical features and histo- pathological results were recorded in a data collection sheet. Statistical analysis was performed by using window based computer software devised with Statistical Packages for Social Sciences (SPSS-17), 95\% confidence limit was taken.

\section{Results}

Table 1: Distribution of Study Population according to MRI and Histopathological Diagnosis $(n=48)$

\begin{tabular}{|l|c|c|}
\hline MRI Diagnosis & Frequency & Percent \\
\hline Pilocytic astrocytoma (Grade-1) & 4 & 8.3 \\
Low grade astrocytoma (Grade-II) & 15 & 31.2 \\
Anaplastic astrocytoma (Grade-III) & 20 & 52.1 \\
Glioblastoma multiformis (Grade-IV) & 9 & 16.7 \\
Total & $\mathbf{4 8}$ & $\mathbf{1 0 0 . 0}$ \\
\hline Histopathological Diagnosis & Frequency & Percent \\
\hline Pilocytic astrocytoma (Grade-I) & 5 & 10.4 \\
Low grade astrocytoma (Grade-II) & 14 & 29.2 \\
Anaplastic astrocytoma (Grade-III) & 21 & 43.8 \\
Glioblastoma multiformis (Grade-IV) & 8 & 16.7 \\
\hline Total & $\mathbf{4 8}$ & $\mathbf{1 0 0 . 0}$ \\
\hline
\end{tabular}

Table 1 shows the distribution of study population according toMRI and Histopathological Diagnosis. The MRI findings are as followed- Pilocytic astrocytoma (Grade-1) is found in 4(8.3\%) cases. Low grade astrocytoma (Grade-II) was found in 15(31.2\%) cases. Anaplastic astrocytoma (Grade-III) was found in 20(52.1\%) cases. Glioblastoma multiformis (Grade-1V) was found in $9(52.1 \%)$ cases.According to histopathological diagnosis, Pilocytic astrocytoma (Grade-1) was found in 5(10.4\%) cases. Low grade astrocytoma (Grade-II) is found in $14(29.2 \%)$ cases. Anaplastic astrocytoma (Grade-III) was found in 21(43.8\%) cases. Glioblastoma multiformis (Grade-1V) was found in 8(16.7\%) cases.

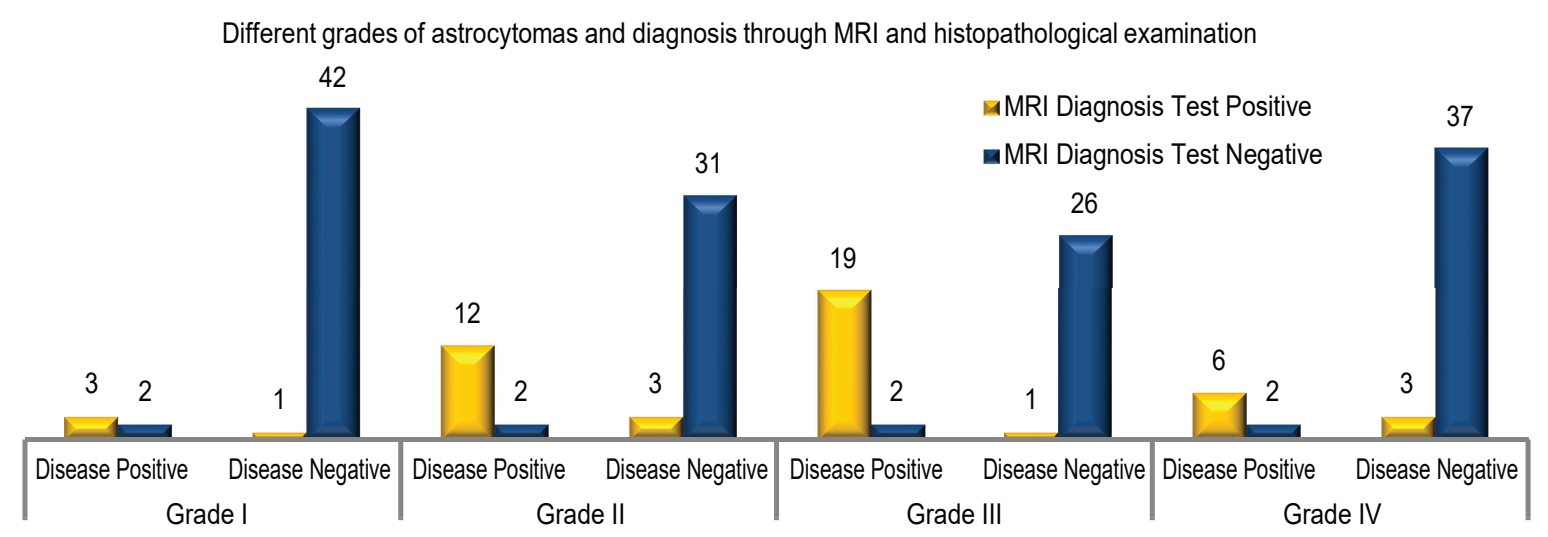

Fig. 1: Comparison of MRI findings with Histopathological Findings during Diagnosis of Different Grades of Astrocytoma $(\mathrm{n}=48)$ 
The Figure 1 shows the comparison of MRI findings with Histopathological Findings during diagnosis of different Gradesof Astrocytoma. In grade I, both histopathological and MRI positive astrocytoma case is found in 3 cases which indicate true positive. Both histopathological and MRI negative astrocytoma case is found in 42 cases which indicate true negative. Histopathological positive but MRI negative case is found in 2 cases which is known as false negative. Histopathological negative but MRI positive case is found in 1 case which is known as false positive.Similarly, in case of Grade II Astrocytoma, true positive cases were 12,31 cases found to be true negative.False negative result found in 2 cases and in 3 casesfound as false positive. In case of Grade IIIastrocytoma, 19 cases were true positive and 26 cases were true negative; 2 cases were false negative and 1 case was false positive. In case of Grade IV Astrocytoma6 cases weretrue positive, 37 cases were true negative, 2 cases were false negative and 3 cases were false positive.

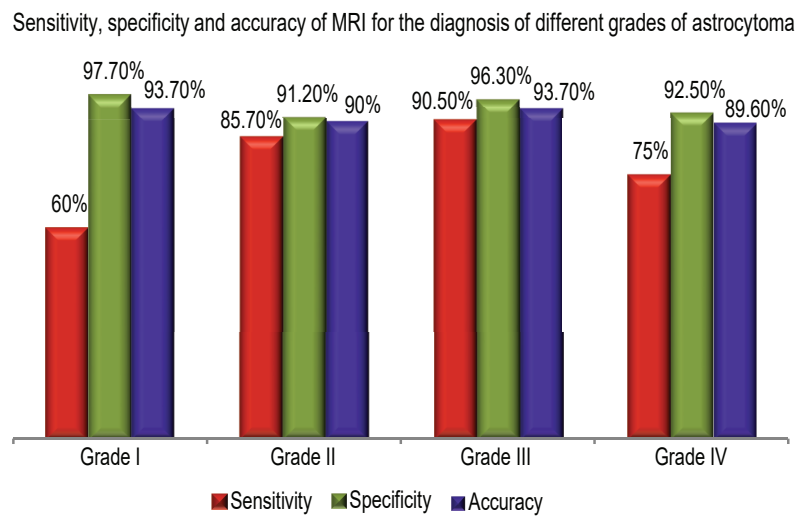

\section{Figure2: Comparison of Validityand Accuracy of MRI for the Diagnosis of Different grade of Astrocytoma}

The sensitivity, specificity, positive predictive value, negative predictive value and accuracy of MRI for the diagnosis of astrocytoma grade I were 60.0\%(95\% CI 46.1-73.9\%), $97.7 \%$ (95\% CI 93.5-101.9\%), $75.0 \%$ (95\% CI 62.7-87.2\%), 95.4\%(95\% CI 89.5-101.3\%) and 93.7\%(95\% CI 86.8-100.6\%) respectively. The sensitivity, specificity, positive predictive value, negative predictive value and accuracy of MRI for the diagnosis of astrocytoma grade II were $85.7 \%$ (95\% CI 56.1-97.2\%), 91.2\% (95\% CI 75.2-97.2\%), 80.0\% (95\% CI 51.4-94.7\%), 93.9\% (95\% CI 78.4-98.9\%) and 90.0\% (95\% CI 81.5-98.49\%) respectively. The sensitivity, specificity, positive predictive value, negative predictive value and accuracy of MRI for the diagnosis of astrocytoma grade IIII were 90.5\% (95\% CI 68.2-98.3), $96.3 \%$ (95\% CI 79.1-99.8\%), $95.0 \%$ (95\% CI 73.0-99.7\%), 92.9\% (95\% CI 75.0-98.7\%) and $93.7 \% \quad(95 \% \quad$ CI $\quad 86.8-100.6 \%)$ respectively. The sensitivity, specificity, positive predictive value, negative predictive value and accuracy of MRI for the diagnosis of astrocytoma grade IV were 75.0\% (95\% CI 62.7-87.2\%), $97.7 \% \quad(95 \% \quad$ CI $\quad 85.5-99.9 \%), \quad 75.0 \% \quad(95 \% \quad$ CI 53.4-80.0\%), $95.4 \%(95 \%$ CI $\quad 88.7-101.1 \%) \quad$ and 93.7\%(95\% CI $81.0-98.2 \%)$ respectively. The highest sensitivity was found in grade III astrocytoma (90.5\%) followed by grade II (85.7\%), grade IV $(75.0 \%)$ and grade I (60.0\%). The highest specificity was found in grade I astrocytoma $(97.7 \%)$ followed by grade III (96.3\%), grade IV (92.5\%) and grade II (91.5\%). The highest Accuracy was found in grade I astrocytoma $(93.7 \%)$ and grade III (93.7\%) followed by grade II (92.5\%) and grade IV $(89.6 \%)$

\section{DISCUSSION}

The comparison of MRI findings with histopathological findings during diagnosis of Grade I Astrocytoma was recorded. Both histopathological and MRI positive astrocytoma case is found in 3 cases were true positive, 42 cases were true negative, 2 cases were false negative and 1 case was falsepositive. The validity of MRI during diagnosis of Grade I astrocytoma was recorded. Thesensitivity, specificity, positive predictive value, negative predictive value and accuracy of MRI for the diagnosis of astrocytoma grade I are $60.0 \%$ (95\% CI 46.1-73.9\%), $97.7 \%$ (95\% CI 93.5-101.9\%), $\quad 75.0 \% \quad(95 \% \quad$ CI $\quad 62.7-87.2 \%)$, $95.4 \%(95 \%$ CI $89.5-101.3 \%)$ and $93.7 \%$ (95\% CI 86.8-100.6\%) respectively.Incase of Grade II astrocytoma, true positive, true negative, false negative and false positive cases were 12, 31, 2 and 3 respectively. The sensitivity, specificity, positive predictive value, negative predictive value and accuracy of MRI for the diagnosis of astrocytoma grade II are $85.7 \%$ (95\% CI 56.1-97.2\%), 91.2\% (95\% CI 75.2-97.2\%), 80.0\% (95\% CI 51.4-94.7\%), 93.9\% (95\% CI 78.4-98.9\%) and 90.0\% (95\% CI 81.5-98.49\%) respectively. Similar findings are reported by Chishty et al ${ }^{7}$, who have recorded $100.0 \%$ sensitivity of MRI in detection of low grade gliomas. The study also recorded similar sensitivity of MRI findings to detect Grade III astrocytoma in correspondence to the study done by Chishty et $\mathrm{al}^{7}$; which for this study was, $90.5 \%$ and for the study of Chishty et $\mathrm{al}^{7}$ it was $95 \%$. Law et $\mathrm{al}^{8}$ observed that PPV and NPV of MRI for determination of a high grade glioma were $86.1 \%$ and $44.1 \%$ respectively which was quite 
consistent with the present study that is, where we found PPV and NPV of MRI for determination of grade IV astrocytomas the $70 \%$ and $95.4 \%$ accordingly.

The highest sensitivity of MRI for the diagnosis of different grade of astrocytoma was found in grade III astrocytoma (90.5\%) followed by Grade II (85.7\%) grade IV (75.0\%) and grade I $(60.0 \%)$. It is interesting that during comparison this result shows that sensitivity of the MRI for the detection of astrocytoma is decreased after increasing of grade of the tumour. Similar to the present findings Ellika et $\mathrm{al}^{9}$ has reported that sensitivity is $85.7 \%$ for different grading of astrocytomas with conventional MRI which is very close to the results of present study. Law et a ${ }^{8}$ found sensitivity of glioma grading ranging from $55.1 \%$ to $83.3 \%$.

During comparing the specificity of MRI for the diagnosis of different grade of astrocytoma we recorded that, the highest specificity was found in grade I astrocytoma (97.7\%) followed by Grade III (96.3\%), grade IV (92.5\%) and grade II (91.5\%). These findings clearly show that the specificity of MRI for the detection of astrocytoma is very high and all are more than $90.0 \%$ which is very conclusive in diagnosis of astrocytoma. Law et $\mathrm{al}^{8}$ observed specificity of MRI in the diagnosis of high grade gliomas was $65 \%$ and in Riemann et $\mathrm{al}^{10}$ series it was $80.0 \%$ in diagnosing low grade gliomas which is in line with the present study. During diagnosis the specificity is very important for the detection of the disease state ${ }^{11-12}$.

During comparing the accuracy of MRI for the diagnosis of different grade of astrocytoma, the highest accuracy was recorded in both grade I astrocytoma (93.7\%) and grade III (93.7\%) followed by grade II (92.5\%) and grade IV (89.6\%). Accuracy of MRI for the detection of astrocytoma is very much effective and in all grade of tumor it was almost $90.0 \%$. This indicates that MRI is a very effectivediagnostic tool for the detection of different grades of astrocytoma. Similar to this result Chishty et $\mathrm{al}^{7}$ found $94.0 \%$ accuracy of preoperative MRI grading of intracranial astrocytomas. Again Riemann et al ${ }^{10}$ found 88.0\% accuracy of contrast enhanced MRI for detecting intracranial astrocytomas which is almost similar to the present study. From the results of present study it is conclusive that MRI scan is a highly accurate and valid modality in the evaluation of different grades of brain tumours.

\section{CONCLUSION}

From the findings of this study it can be concluded thatMRI is an effective tool for the diagnosis of different grades of brain tumours, for this study which is astrocytomas. Sensitivity, specificity and accuracy of MRI for the diagnosis of astrocytoma are high in different grades of astrocytoma thus its validity is highly dependable.

\section{REFERENCES}

1. Leeds NE, Kieffer SA. Evolution of Diagnostic Neuroradiology from 1904 to 1999. Radiology 2000; 217(2):309-18.

2. Haaga JR, Kieffer SA, Chang JK, Lanzieri CF, Gilkeson RC. Intracranial Neoplasms' Computed Tomography and Magnetic Resonance Imaging of the whole body, Mosby, USA; 4th ed. 2003;1:59

3. Geets X, Daisne JF, Arcangeli S, Coche E, De Poel M, Duprez T, Nardella G, Grégoire V. Inter-observer variability in the delineation of pharyngo-laryngeal tumor, parotid glands and cervical spinal cord: comparison between CT-scan and MRI. Radiotherapy and oncology. 2005;77(1):25-31

4. Boss A, Bisdas S, Kolb A, Hofmann M, Ernemann U, Claussen CD, Pfannenberg C, Pichler BJ, Reimold M, Stegger L. Hybrid PET/MRI of intracranial masses: initial experiences and comparison to PET/CT. Journal of Nuclear Medicine. 2010; 51(8):1198-205

5. Allen CMC, Lueck CJ, Dennins M. Davidson's Principles and Practice of Medicine.20th ed.Churchill Living Stone,India, 2006;236

6. Komotar RJ, Mocco J, Jones JE, Zacharia BE, Tihan T, Feldstein NA, Anderson RC. Pilomyxoid astrocytoma: diagnosis, prognosis, and management. Neurosurgical focus. 2005;18(6):1-4

7. Chishty IA, Rafique MZ, Hussain M, Akhtar W, Ahmed MN, Sajjad Z, Ali SZ. MRI characterization and histopathological correlation of primary intra-axial brain glioma. JLUMHS. 2010;9(02):64

8. Law M, Yang S, Wang H, Babb JS, Johnson G, Cha S, Knopp EA, Zagzag D. Glioma grading: sensitivity, specificity, and predictive values of perfusion MR imaging and proton MR spectroscopic imaging compared with conventional MR imaging. American Journal of Neuroradiology. 2003;24(10):1989-98

9. Ellika SK, Jain R, Patel SC, Scarpace L, Schultz LR, Rock JP, Mikkelsen T. Role of perfusion CT in glioma grading and comparison with conventional 
MR imaging features. American Journal of Neuroradiology. 2007;28(10):1981-7

10. Riemann B, Papke K, Hoess N, Kuwert T, Weckesser M, Matheja P, Wassmann H, Heindel W, Schober O. Noninvasive Grading of Untreated Gliomas: A Comparative Study of MR Imaging and 3-(Iodine 123)-L-a-methyltyrosine SPECT 1. Radiology. 2002;225(2):567-74

11. Roth J, Roach ES, Bartels U, Jóźwiak S, Koenig MK, Weiner HL, Franz DN, Wang HZ. Subependymal giant cell astrocytoma: diagnosis, screening, and treatment. Recommendations from the International Tuberous Sclerosis Complex Consensus Conference 2012. Pediatric Neurol 2013;49(6):439-44

12. Harrer JU, Parker GJ, Haroon HA, Buckley DL, Embelton K, Roberts C, Balériaux D, Jackson A. Comparative study of methods for determining vascular permeability and blood volume in human gliomas. Journal of Magnetic Resonance Imaging. 2004;20(5):748-57 